\title{
Analysis of Inductive Multiport Microwave Devices Employing a Novel Double Parallel Plate Approach
}

\begin{tabular}{|r|l|}
\hline Journal: & IET Microwaves, Antennas \& Propagation \\
\hline Manuscript ID: & MAP-2007-0132 \\
\hline Manuscript Type: & Research Paper \\
\hline Complete List of Authors: & $\begin{array}{l}\text { ALVAREZ-MELCON, ALEJANDRO; TECHNICAL UNIVERSITY OF } \\
\text { CARTAGENA, AND COMMUNICATION TECHNOLOGIES } \\
\text { Perez-Soler, Javier; Technical University Cartagena, Information } \\
\text { Technologies } \\
\text { Quesada-Pereira, Fernando; Technical University Cartagena, } \\
\text { Information Technologies } \\
\text { Gomez Diaz, Juan-Sebastian; Technical University Cartagena, } \\
\text { Information Technologies }\end{array}$ \\
\hline Keyword: & $\begin{array}{l}\text { FILTERS, INTEGRAL EQUATIONS, MICROWAVE TECHNIQUES, } \\
\text { NUMERICAL ANALYSIS, WAVEGUIDE FILTERS }\end{array}$ \\
\hline &
\end{tabular}

\section{(5) ScholaronE" \\ Manuscript Central}




\title{
Analysis of Inductive Multiport Microwave Devices Employing a Novel Double Parallel Plate Approach
}

\author{
F. J. Pérez Soler, F. D. Quesada-Pereira, J. S. Gómez-Díaz and A. Alvarez-Melcon \\ Technical University of Cartagena, Cuartel de Antigones s/n, Cartagena, ES-30202, Spain, \\ Phone: +34-968-32 6532. Fax: +34-968-32 5338. E-mail: fjps@alu.upct.es
}

June 6, 2007

Key Words: Integral Equations, Green's Functions, Waveguide components, Numerical analysis, Dielectric objects

\begin{abstract}
In this paper we present a novel surface Integral Equation (IE) approach for analyzing inductive microwave devices with several parallel input-output ports containing inductive metallic and dielectric obstacles. The technique decomposes the main problem into three subproblems, employing different kinds of Green's functions. One of the subproblems uses the classical Green's functions of an infinite unbounded medium with the constitutive parameters of the dielectric obstacles. A novel point of the technique is the formulation of the two other subproblems with two different, 90-degrees rotated parallel plate regions. In these regions the parallel plate waveguide Green's functions are used to simplify the modeling of the excitation problem. The second novel aspect of the work is in the treatment of these Green's functions, in order to smooth their behavior, and to improve their convergence. Several numerical results are presented to demonstrate the usefulness of the algorithms proposed. Also, several results for inductive microwave devices are presented to show the practical value of the approach.
\end{abstract}

\section{Introduction}

The study of microwave waveguide devices has been a matter of interest for the last decades. A wide class of inductive waveguide devices finds application in many high power satellite and mobile communication systems. This has forced the necessity for the development of CAD tools that allow the analysis and design of different kinds of inductive waveguide components in a fast and efficient way. For this purpose, several different methods have been studied, including finite element methods ([1]), modal analysis techniques ([2]), and Integral Equation (IE) approaches employing different formulations $([3,4,5])$. In [6], a surface IE formulation was proposed to study inductive structures including metallic and dielectric objects inside the waveguide. The technique combines the parallel plate waveguide (PPW) Green's functions and the so-called PMCHWT [7] formulation for the treatment of dielectric objects. The main disadvantage of that approach is that all elements of the structure must share 
the same base waveguide. Consequently, useful microwave devices such as T-junctions, bifurcations, bends or diplexers cannot be analyzed.

In order to take these structures into account, another method was proposed in [8], which allowed to study inductive devices with several arbitrarily oriented ports. In that work the Surface Equivalence Principle [9] was also applied, isolating the excitation problem by means of a ground plane placed at the excitation port. The whole problem was divided in two simpler problems. The first one was focused on the excitation port, and used the PPW Green's functions. The second problem was formulated in the internal structure, employing classic spatial image theory for the Green's functions. Besides, that second problem could be accelerated by using the Fast Multipole Method (FMM) technique [10], since it was based on the free-space Green's functions. By using that approach, a wide class of different multiport microwave devices could be analyzed, provided that the input-output ports were not aligned along parallel planes. This is because the ground plane used to model the ports introduces a shortcircuit into the ports aligned with the current active port. Due to this limitation, important devices such as multiplexers, bifurcations, or power dividers, which have several ports aligned on the same plane, could not be directly treated with the technique.

In this paper, we present a novel method that takes profit of the ideas presented in the two previous works, and allows to successfully analyze multiport devices having the ports aligned on two different planes. Using again the Surface Equivalence Principle, the main problem is now decomposed in three subproblems. The first one tackles the excitation port, employing the PPW Green's functions. The second problem is related to the internal circuit of the structure, which can contain different kinds of metallic and dielectric inductive posts. This problem also employs PPW Green's functions, but inside a waveguide rotated 90 degrees with respect to the port waveguides. Finally, a third equivalent problem is formulated inside the dielectric posts, employing the Green's functions of an infinite unbounded homogenous medium with the constitutive parameters of the posts.

For the implementation of the technique, a key issue is the calculation of the relevant PPW Green's functions. For this calculation, a combination of two efficient acceleration techniques, namely the Kummer [11] and the Ewald [12] methods are employed, as introduced in [6]. In the rotated parallel plate waveguide of the second subproblem, the distance between the plates can be considerably large, in order to fit electrically large structures. Consequently, the convergence properties of the two previous techniques are for the first time explored under this new situation. As expected, results show that more terms are needed in the series, as the parallel plate width increases.

To limit the impact in the efficiency when the separation of the parallel plates is electrically large, a new treatment of the PPW Green's functions is proposed. The idea is extracting the fast variations from the PPW Green's functions, so that the reminder can be integrated with very few integration points. In this way, the number of evaluations required for the time consuming PPW Green's functions is reduced. In a first step, the strategy extracts the singularity of the Green's functions as a spatial domain source term. The remainder behaves smoothly, except for source points very close to one of the parallel plate walls. To cope with this situation, a second step is applied by extracting a spatial image term with respect to the closest wall to the source point. The two spatial domain terms are extracted from the PPW spectral series, in the form of simple Hankel functions. Since they contain the fast variations of the Green's functions, more points are needed to integrate them. However, the evaluation of these terms in the form of Hankel functions is fast, as compared to the original slowly 
convergent PPW series.

In the paper we present a careful study on the convergence of the series, and on the behavior of the Green's functions after the extraction of the spatial domain terms. Finally, some results for practical inductive waveguide devices are presented. The comparisons with measurements and with a finite element method prove the accuracy and efficiency of this new double parallel plate strategy.

\section{Theoretical Outline}

The problem under study is presented in Fig. 1. It consists of an arbitrarily shaped cavity which contains metallic and dielectric inductive posts, surrounded by $N_{p}$ input/output ports aligned along two parallel planes.

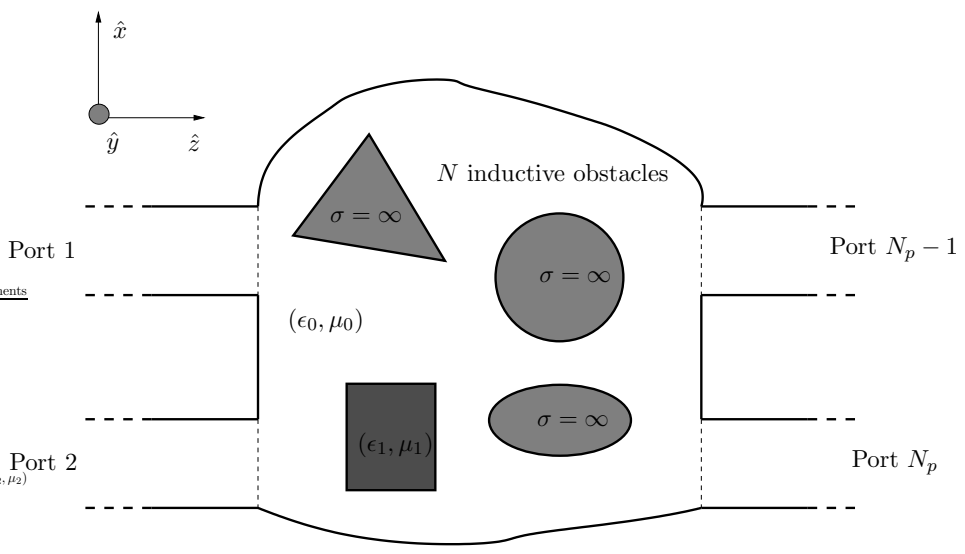

Figure 1: Inductive microwave problem under study, which contains metallic and dielectric objects, and $N_{p}$-exciting ports.

In order to analyze this structure, the Surface Equivalence Principle is applied to the original problem in Fig. 1, obtaining three different simpler problems. The first problem is sketched in Fig. 2, and corresponds to the input-output waveguides connected to each port. Inside each waveguide the only existing source is a magnetic current density $2 \vec{M}_{p n}$, located at the connecting boundary between the port $(n)$ and the main cavity of the structure (surface $S_{p n}, n=1,2 \cdots N_{p}$ ). This magnetic current density radiates by means of the PPW Green's functions of the corresponding waveguide used to define the port $(n)$. The value of the current is doubled due to the application of the image theory to a horizontal magnetic current in the presence of a perfectly conducting plane, which separates the port region from the main structure. The excitation for this problem generally consists in the fundamental $T E_{10}$ mode, incident to the port exciting the circuit.

The second equivalent problem is formulated inside the main cavity of the circuit, which contains the metallic and dielectric posts, as shown in Fig. 3. This problem is separated from the first problem by means of two parallel infinite ground planes, located at the boundaries which connect the main structure to the aligned input/output ports. The two parallel infinite ground planes actually form another parallel plate region, but with an orthogonal orientation with respect to the waveguides defined at the input/output ports. Inside this second problem we consider several sources. First, we have to consider the magnetic current densities $-\vec{M}_{p n}$ located at each port boundaries $\left(n=1,2 \cdots N_{p}\right)$. Second, we consider the electric current density $\left(\vec{J}_{c}\right)$ induced on the conducting surfaces of the metallic 

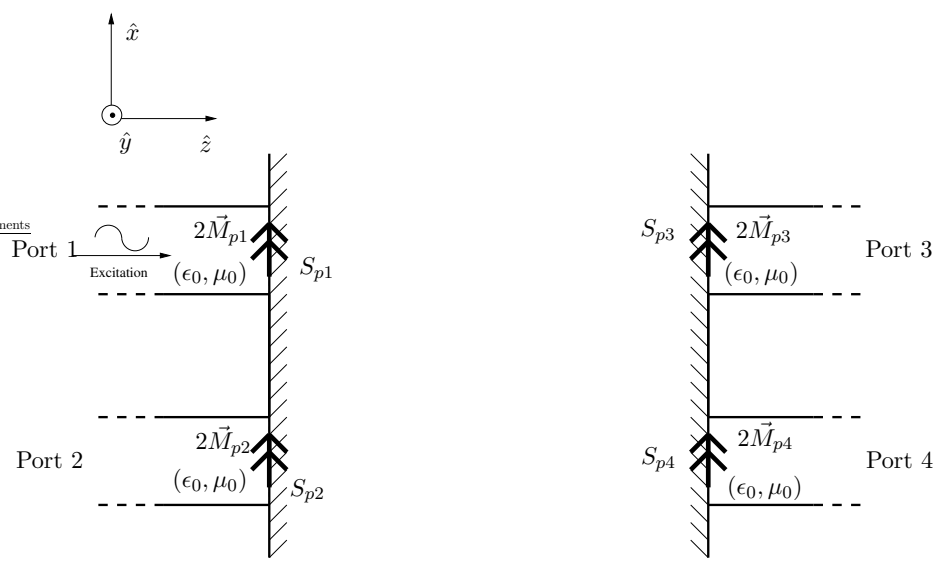

Figure 2: First equivalent problem external to the main cavity of the circuit, where magnetic current densities are defined to model each port. The figure shows the particular case of $N_{p}=4$.

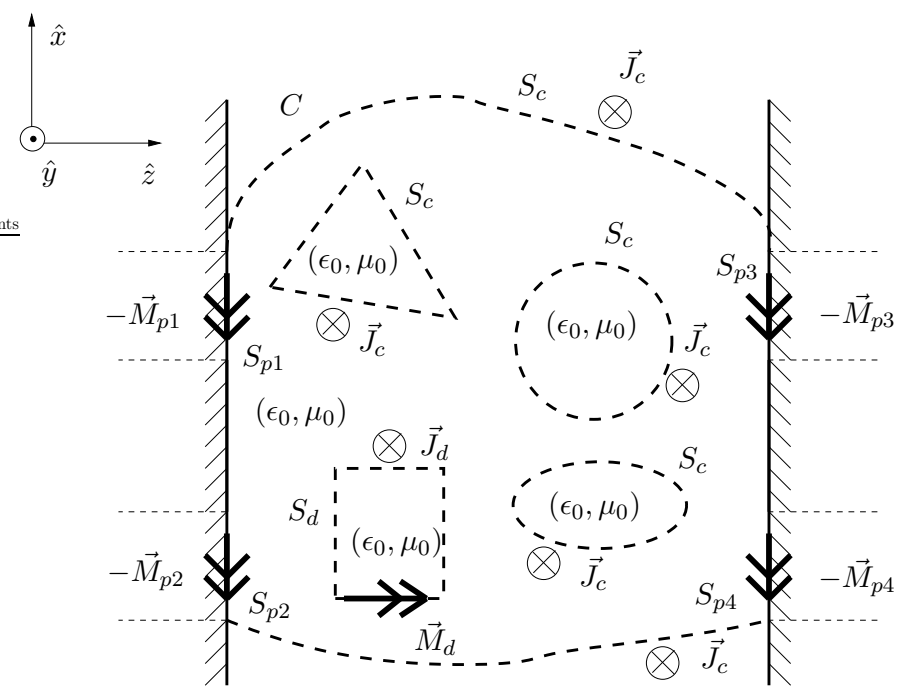

Figure 3: Second equivalent problem corresponding to the core of the structure. Electric and magnetic current densities are considered on the surfaces of the different posts.

posts. Finally, we must also consider the corresponding equivalent electric $\left(\vec{J}_{d}\right)$ and magnetic $\left(\vec{M}_{d}\right)$ current densities defined on the surfaces of the dielectric posts. All these sources radiate inside the $90^{\circ}$ rotated parallel plate waveguide shown in Fig. 3 .

Finally, the last equivalent problem corresponds with the internal regions of the dielectric posts (see Fig. 4). In this case, the only sources are the electric and magnetic current densities, $-\vec{J}_{d}$ and $-\vec{M}_{d}$, with opposite directions with respect to the previous problem. This time the currents radiate with the Green's functions of an infinite unbounded homogeneous medium with the constitutive parameters of the dielectric post, $\left(\epsilon_{1}, \mu_{1}\right)$. It should be remarked that the different electric current densities are oriented along the $\hat{y}$ axis, because this is the direction of the electric field excited in the treated inductive problems. For the same reason, the magnetic currents are oriented following the contour of the different surfaces on which they are defined $((x, z)$-plane, see Fig. 1). Between these three equivalent problems, the tangential electric and magnetic fields must be continuous. This boundary condition, together with the imposition of the nullity of the tangential electric fields on the conducting surfaces, allows obtaining a set of coupled integral equations. The details of the integral equations 


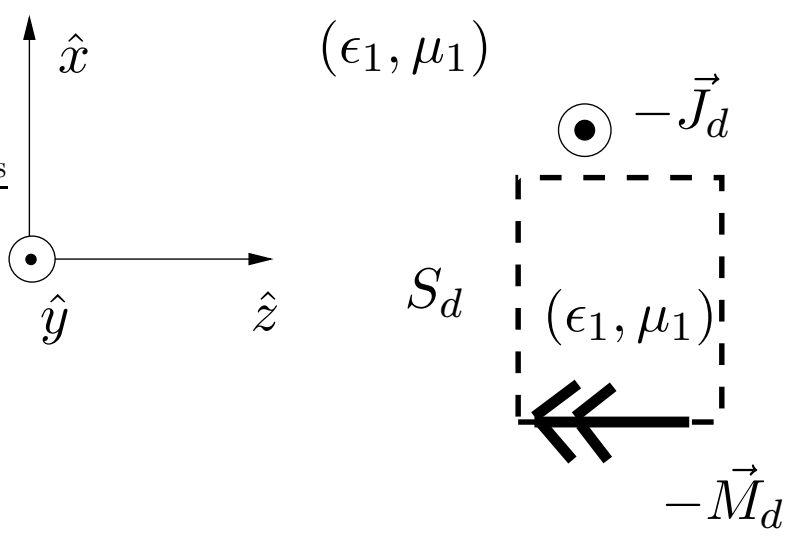

Figure 4: Third equivalent problem, formulated inside the internal regions of the dielectric posts.

formulated for general metallic and dielectric objects are given in the Appendix.

\subsection{Effect of the size of the PPW width in the convergence of the Green's functions}

As it has been described before, the proposed method uses the PPW Green's functions for two different waveguides in the first and the second subproblems. In the first problem, the parallel plate waveguides of the input/output ports are used (see Fig. 2). Since the parallel plate for the second problem must contain the core of the structure (see Fig. 3), its width will be typically much larger in electrical terms than the one corresponding to an ordinary excitation port. This fact directly affects the calculation of the corresponding Green's functions, since the relevant series might exhibit slower convergence rates. In fact, we have observed that when the width of the PPW increases, the number of terms needed in the series to maintain a given accuracy is larger. This is because a certain number of higher order modes are propagating inside the wider waveguide, as compared to the narrower PPW used in the input-output ports, where only the dominant mode propagates.

In order to illustrate the importance of this fact, a convergence study is presented for the Green's functions calculated by combining both Kummer and Ewald methods [6]. Let us consider first the Kummer transformation. This method basically consist in extracting the asymptotic part of the spectral domain modal series, so that the dynamic part converges faster. The asymptotic series can then be summed up in closed form, using the technique presented in [11]. For the numerical test we will consider a source located at the center of the waveguide $x^{\prime}=a / 2$, whereas the observation point will be taken at a distance of $\left|z-z^{\prime}\right|=0,\left|x-x^{\prime}\right|=0.01$, from the source point. The observation point, then, corresponds to point $A$ shown in Fig. 5.

Fig. 6 shows the relative error obtained in the calculation of the dyadic component $G_{A}^{y y}$ of the PPW Green's functions and its derivative $\partial G_{A}^{y y} / \partial x$, as a function of the number of modes employed for the summation of the spectral series. The study has been performed for three different waveguide widths $(a=0.75 / \lambda, a=1.75 / \lambda$ and $a=2.75 / \lambda)$. It can be observed in Fig. 6 (a) that the number of modes needed to obtain a relative error lower than $10^{-4}$ in the calculation of $G_{A}^{y y}$ for the three widths are respectively 25,50 and 75 . For the derivative $\left(\partial G_{A}^{y y} / \partial x\right)$, Fig. 6(b) shows a similar behavior, but with a slower convergence rate. In this case we need 100, 225 and 350 modes for achieving the same precision of $10^{-4}$, for the three different studied waveguide widths. The slower convergence rate for 


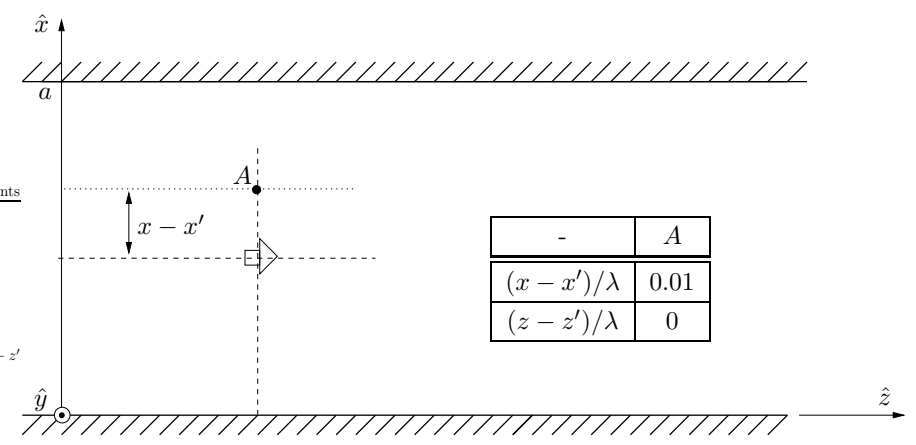

Figure 5: Position of the source and observation points inside a parallel plate waveguide, for the proposed convergence study.

the spatial derivative is a known fact of the spectral domain series [6]. However, the important point

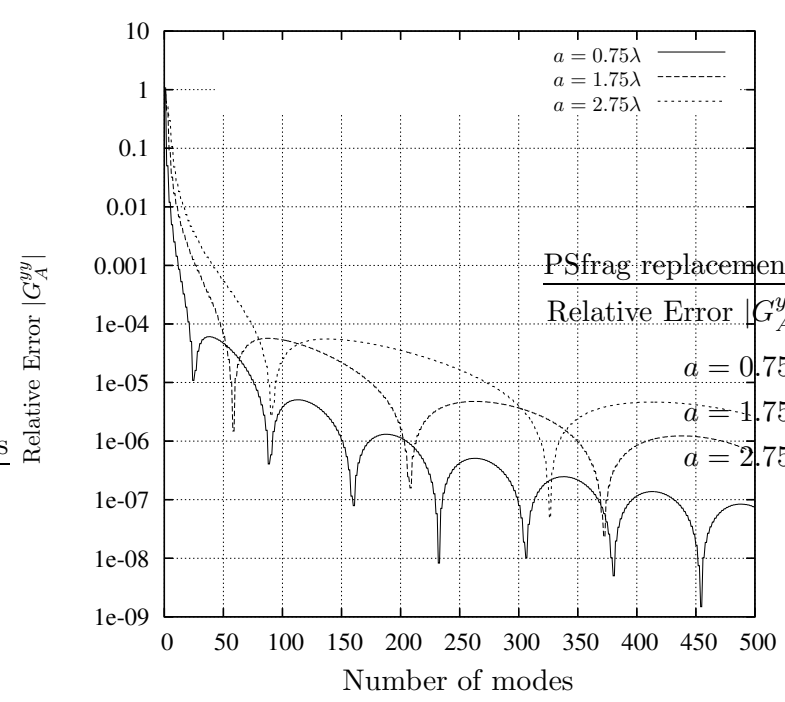

(a) Convergence of $G_{A}^{y y}$.

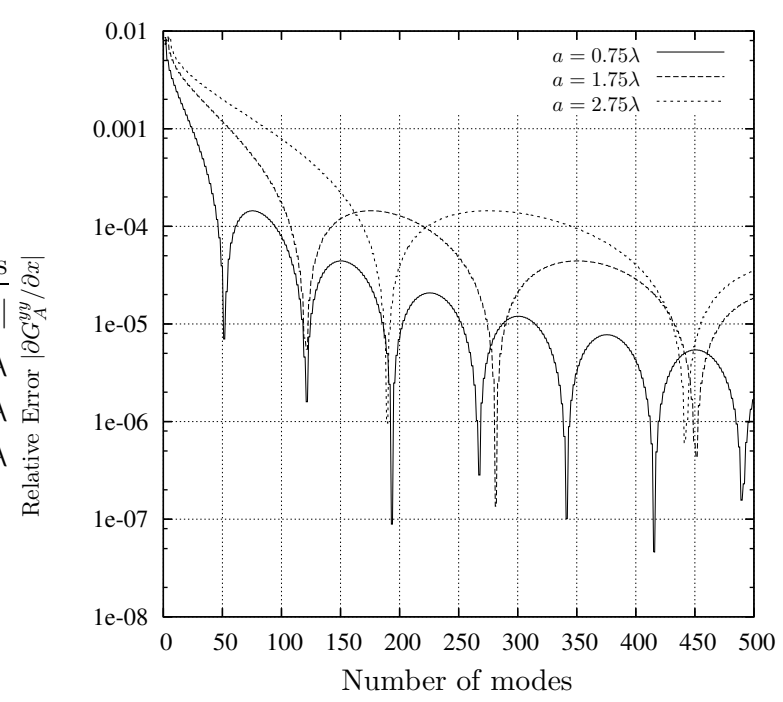

(b) Convergence of $\partial G_{A}^{y y} / \partial x$.

Figure 6: Green's functions convergence employing the Kummer transformation for three different waveguide widths $(a=0.75 / \lambda, a=1.75 / \lambda$ and $a=2.75 / \lambda)$, when the observation point is placed at position $A$ of Fig. 5 .

now is to see that the convergence in any case is slower when the parallel plate width increases.

To complete the convergence analysis, in Fig. 7 we present the same study as before but for the Ewald method. This technique accelerates the convergence of the series by a mathematical transformation that separates the original series in two different terms (one spectral and the other spatial). The splitting of the original series is controlled by means of the so-called splitting parameter as introduced in ([12]). In this case the convergence depends on the values of the parameters $M, P$ also described in [12]. Attending to Fig. 7(a), in order to maintain a relative error lower than $10^{-4}$ in the calculation of $G_{A}^{y y}$, we need $M=P=2, M=P=4$ and $M=P=7$ for the cases $a=0.75 / \lambda, a=1.75 / \lambda$ and $a=2.75 / \lambda$, respectively. If we fix our attention to the derivative, a similar convergence behavior is obtained. In this case we need $M=P=2, M=P=6$ and $M=P=8$ for each one of the previous widths. As a difference with respect to the Kummer transformation, the number of terms required in the Ewald method does not change significantly for the derivatives as compared to the ordinary Green's functions components. However, the important point now is to note that, again, the number of terms needed in the Ewald technique also increases for larger plate separations. 
If we compare the performances of the Kummer and the Ewald technique, we can observe that the number of terms $M=P$ in the Ewald technique is smaller than the number of modes in the Kummer method, for the different values of the waveguide widths. As a disadvantage, due to the presence of the complementary error functions of complex arguments inherent to the formulation of the Ewald method ([12]), the involved calculations require higher computational cost. Consequently, the best

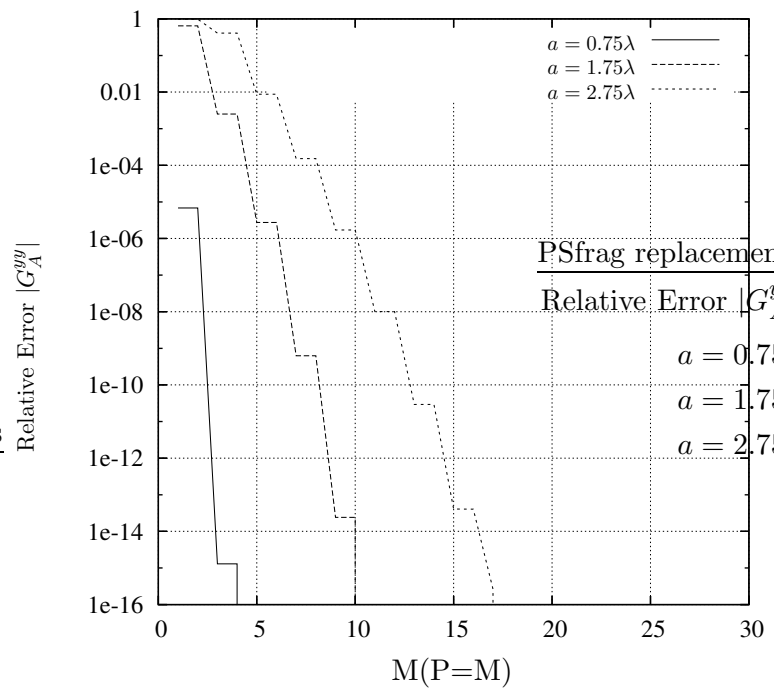

(a) Convergence of $G_{A}^{y y}$.

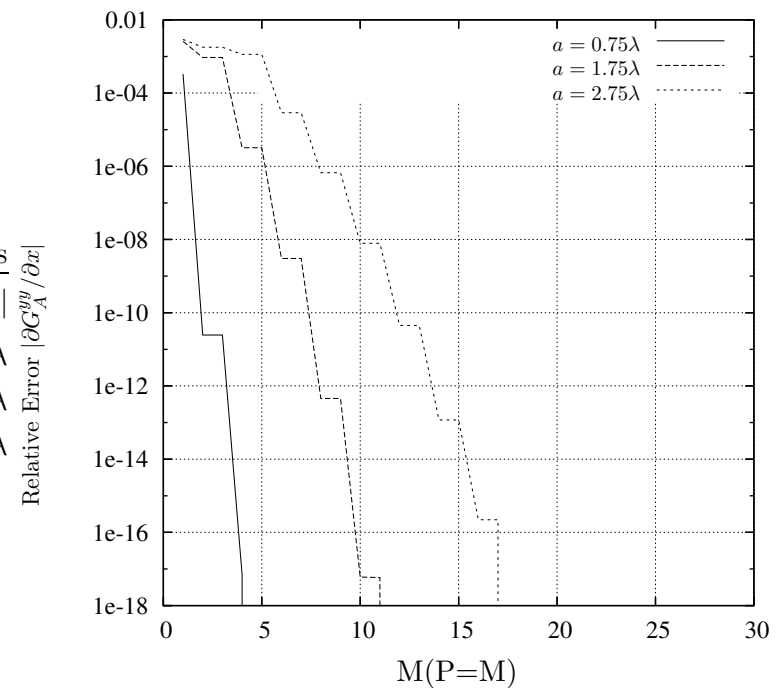

(b) Convergence of $\partial G_{A}^{y y} / \partial x$.

Figure 7: Green's functions convergence employing the Ewald method for three different waveguide widths $(a=0.75 / \lambda, a=1.75 / \lambda$ and $a=2.75 / \lambda)$, when the observation point is placed at position $A$ of Fig. 5 .

practice is to combine both techniques for an increased efficiency, as reported in [6].

As it has been shown, the number of terms employed in the Kummer and Ewald techniques must be increased for large parallel plate widths. This behavior could represent a problem for the double parallel plate approach presented in this paper. In fact, parallel plates with large widths might be needed to fit electrically large devices (see Fig. 3). To limit the impact of this behavior in the computational cost associated to the analysis of practical inductive circuits, an alternative treatment of the Green's functions is now proposed.

Since the convergence of the Green's functions is slower when the width of the parallel plate increases, the idea is to limit the number of evaluations required in the associated series. This can be done if the part of the Green's functions represented by the slowly convergent series exhibits very smooth behaviors. Then, this reminder can be integrated during the calculation of the MoM matrix with a reduced number of points, therefore limiting the number of evaluations of the series. To obtain a series representation of a very smooth function, the basic idea is to extract the source term, formulated as a simple Hankel function, from the original slowly convergent series:

$$
G_{R_{0}}=\sum_{n=0}^{\infty} V_{n}(z) f_{n}(x) g_{n}\left(x^{\prime}\right)-H_{0}^{(2)}\left(k \sqrt{\left(x-x^{\prime}\right)^{2}+\left(z-z^{\prime}\right)^{2}}\right)
$$

In above equation, the series represents the original parallel plate Green's function, and the definition of the terms $V_{n}(z), f_{n}(x)$ and $g_{n}\left(x^{\prime}\right)$ can be found in [6]. The argument of the Hankel function of the substracted source term contains the propagation constant $(k)$ and the spatial distance between 
source and observation points. It is important to note that the new reminder definition $\left(G_{R_{0}}\right)$ is also formulated with a slowly convergent series. However, this reminder behaves very smoothly, even close to the singularity.

To illustrate the concept, we present in Fig. 8 the magnitude of the $G_{A}^{y y}$ component in a parallel plate of width $a=4.11 \lambda_{0}$ at a frequency of $12 \mathrm{GHz}$. The source point is located at $x^{\prime}=0.5 a$, and the observation point is varied along the vertical line passing through the source point $\left(\left|z-z^{\prime}\right|=0\right)$. We observe a sharp peak if the observation point is close to the source point, due to the singular behavior of the Green's functions. In the same figure we present the reminder $\left(G_{R_{0}}\right)$ defined in equation (1). We can clearly observe that the singularity is effectively eliminated, presenting a very smooth behavior.

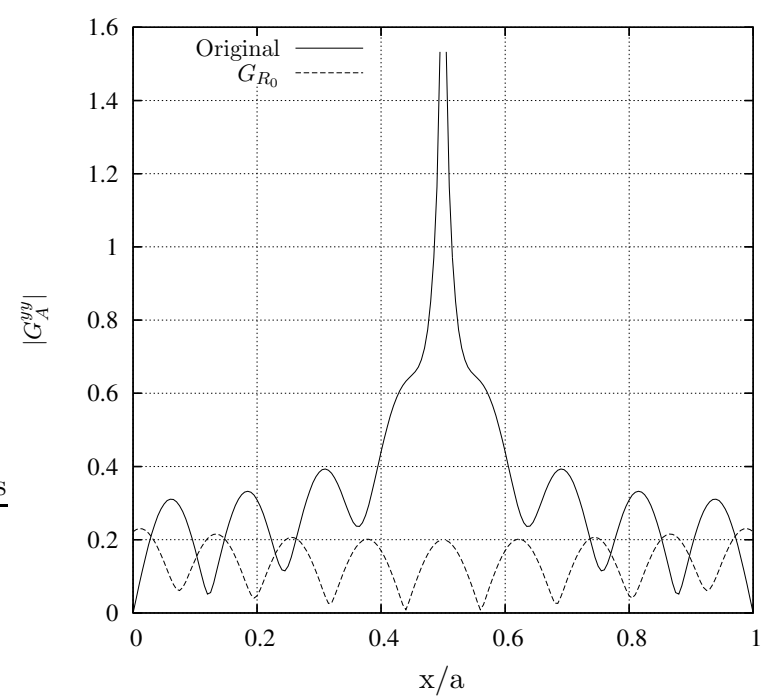

Figure 8: Magnitude of $G_{A}^{y y}$ at the frequency of $12 \mathrm{GHz}$ for a source point located at $x^{\prime}=$ $0.5 a$. The observation point is varied along a vertical line passing through the source point. The definition of the reminder term $\left(G_{R_{0}}\right)$ is also shown.

This simple extraction procedure of the source term is effective when the source point is not close to one of the walls of the waveguide. On the contrary, if the source point is close to one of the walls, the influence of this wall will cause a fast variation of the Green's functions, even if the source term is extracted. To demonstrate this fact we present in Fig. 9 the derivative $\left(\partial G_{A}^{y y} / \partial x\right)$ in the same parallel plate as before, but when the source is placed close to the bottom wall $\left(x^{\prime}=0.002 a\right)$. In this case, we observe a very fast variation due to the presence of the source close to the wall. In the same figure we also present the new reminder $\left(G_{R_{0}}\right)$ for this situation. Even though the source is extracted, a fast variation is observed due to the presence of the wall. This fast variation could be a problem during the calculation of the MoM matrix elements for source cells close to the wall, since more points will be required to achieve convergence in the associated integrals.

The solution to this problem can be found if we think of the original parallel plate Green's functions in terms of the spatial images series. If the source point is close to one of the walls, the first spatial image of the infinite series approaches closer to the wall. The proximity of this spatial image is responsible for the fast variation of the Green's function, even if the source term is extracted. The idea then is to define a new reminder without the source term and without the first spatial image with 
respect to the closest wall to the source point, in the following way:

$G_{R_{1}}=\sum_{n=0}^{\infty} V_{n}(z) f_{n}(x) g_{n}\left(x^{\prime}\right)-H_{0}^{(2)}\left(k \sqrt{\left(x-x^{\prime}\right)^{2}+\left(z-z^{\prime}\right)^{2}}\right)-H_{0}^{(2)}\left(k \sqrt{\left(x-x^{\prime}\right)^{2}+\left[z-\left(z^{\prime}+s_{g} 2 d\right)\right]^{2}}\right)$

In (2), the first two terms are the same as in (1). The contribution of the first spatial image is obtained by considering the distance $(d)$ between the source and the closest plate. The sign $s_{g}$ takes the value $(+1)$ or $(-1)$ if the source is closer to the upper or to the lower plate, respectively. It can be seen that this new term is essentially equal to the source term for small values of $(d)$, and the necessity of its cancellation is therefore justified. It is important to notice that the next image in the spatial series will appear at a distance of $(2 a)$ with respect to the position of the source point. Therefore, the influence of this second spatial image will not contribute, in any case, to fast variations inside the new defined reminder in equation (2).

To demonstrate that this technique is indeed effective, we also present in Fig. 9 the behavior of the second reminder term $\left(G_{R_{1}}\right.$ as defined in equation (2)), calculated in the same situation as before. We observe that the fast variations are completely eliminated, leaving a reminder with a very smooth behavior even for observation points close to the waveguide walls. Once the fast variations

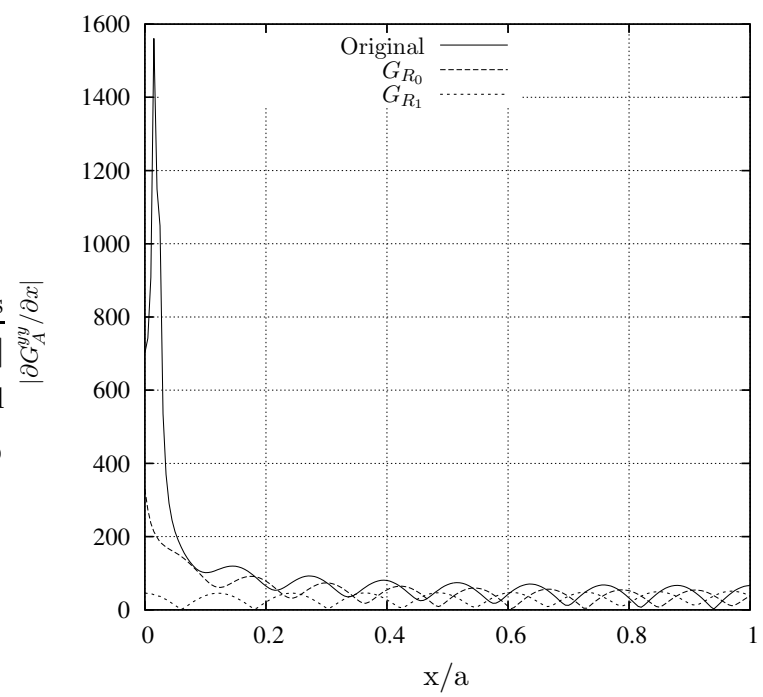

Figure 9: Magnitude of $\partial G_{A}^{y y} / \partial x$ in the same parallel plate as in Fig. 8, when the source is placed at $x^{\prime}=0.002 a$. The reminder terms $G_{R_{0}}$ and $G_{R_{1}}$ are also shown for comparison. Frequency of the analysis is $12 \mathrm{GHz}$.

are extracted from the series representation of the Green's functions, it is possible to integrate the reminder $\left(G_{R_{1}}\right)$ employing very few integration points [13] during the calculation of the MoM matrix. Also note that the subtracted terms, that contain the fast variations, can be integrated separately with more integration points. The integration of these terms requires a proper treatment of the singularity, following standard techniques [9]. Although more points are needed for these terms, they are formulated with standard Hankel functions, and can be computed very fast. The efficient calculation of these terms is possible since no convergence issues are related to them. All convergence issues are now translated to the remainder $G_{R_{1}}$, presenting a very smooth behavior. 


\section{Results}

In this section we will present the analysis of some practical microwave devices employing the new proposed approach. The first example is a waveguide diplexer for satellite applications shown in Fig. 10(a). A total of 442 basis functions have been employed for the expansion of the unknown current densities induced on the ports and on the conducting surfaces. If the Kummer transformation is applied, 200 modes are needed in order to obtain good convergence in the calculation of the PPW Green's functions of the second equivalent problem, when the observation points are placed near the source. For the Ewald method, the values of the parameters $M=P$ have been set to 13 . These values show the computational effort needed in the calculation of the Green's functions of the second problem, due to the electrically large separation of the parallel plates (which varies between $3.15 \lambda$ and $3.85 \lambda$ for the frequency range of the analysis). Due to this fact, it is essential to limit the number of evaluations of the series during a practical analysis.

In Fig. 10(b) we present the results obtained with the novel approach, and we compare them with results evaluated with the commercial tool HFSS ${ }^{\circledR}$. Measured results are also included, showing the accuracy and validity of the new proposed technique. If the direct Green's functions are used in the analysis, the proposed technique takes 58 seconds per frequency point. This is because a slowly convergent series need to be calculated close to the singularity, where more integration points are required. If the proposed Green's functions decomposition is used, the new software tool takes only 34 seconds per frequency point. In this case, we have verified that only one integration point is required for each source cell, to correctly integrate the reminder term $G_{R_{1}}$ of equation (2). To obtain a similar accuracy in $\mathrm{HFSS}^{\circledR}, 24$ minutes per frequency point were needed to complete the analysis, on a standard PC with $3.2 \mathrm{GHz}$ CPU processor.

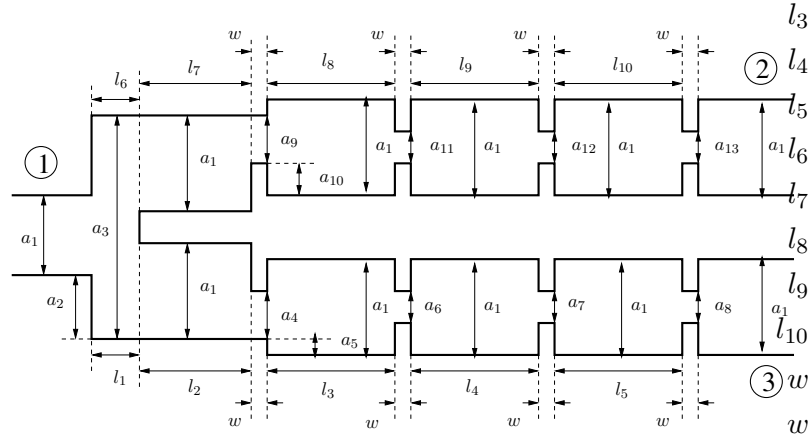

(a) Geometry of the diplexer.

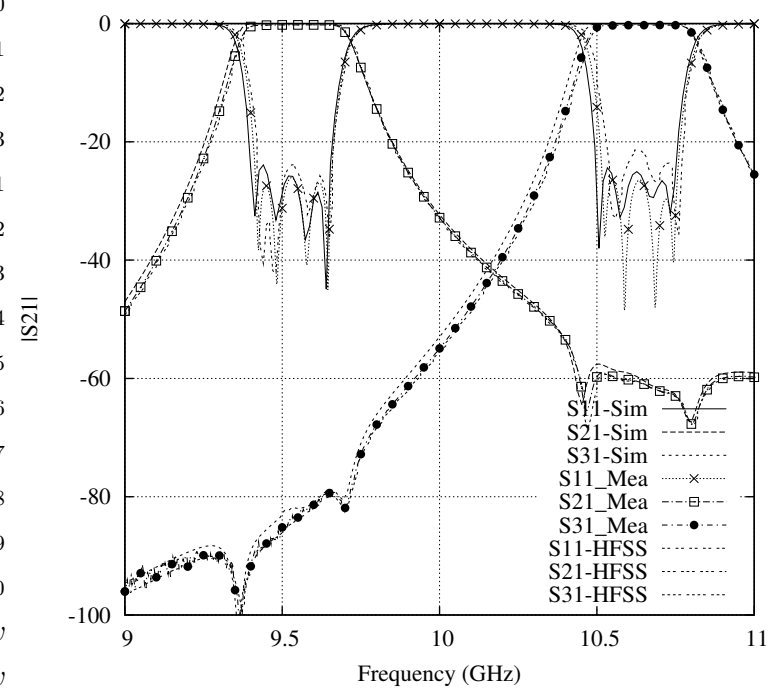

(b) Scattering parameters of the diplexer.

Figure 10: Geometry and scattering parameters of a waveguide diplexer. Dimensions are: $a_{1}=22.86 \mathrm{~mm}, a_{2}=12.95 \mathrm{~mm}, a_{3}=47.72 \mathrm{~mm}, a_{4}=8.29 \mathrm{~mm}, a_{5}=7.285 \mathrm{~mm}$, $a_{6}=6.13 \mathrm{~mm}, a_{7}=6.715 \mathrm{~mm}, a_{8}=10.67 \mathrm{~mm}, a_{9}=9.34 \mathrm{~mm}, a_{10}=6.76 \mathrm{~mm}$, $a_{11}=7.11 \mathrm{~mm}, a_{12}=7.76 \mathrm{~mm}, a_{13}=11.94 \mathrm{~mm}, l_{1}=4.6 \mathrm{~mm}, l_{2}=12.11 \mathrm{~mm}$, $l_{3}=16.12 \mathrm{~mm}, l_{4}=16.58 \mathrm{~mm}, l_{5}=14.904 \mathrm{~mm}, l_{6}=4.6 \mathrm{~mm}, l_{7}=15.46 \mathrm{~mm}$, $l_{8}=19.32 \mathrm{~mm}, l_{9}=19.835 \mathrm{~mm}, l_{10}=17.735 \mathrm{~mm}$, and $w=2.0 \mathrm{~mm}$. 
The next example is a rectangular cavity resonator with a dielectric post placed at the center, as shown in Fig. 11(a). The structure has been caracterized with 150 basis functions. The convergence of the PPW Green's functions is achieved by employing 30 modes for the Kummer transformation, and $M=P=5$ for the Ewald method. In this case the convergence is faster, since the separation of the parallel plate walls, needed to fit the structure, is smaller (between $1.5 \lambda$ and $2.0 \lambda$ for the frequencies under consideration). Fig. 11(b) shows the transmission parameter as compared to results provided by HFSS ${ }^{\circledR}$, and by another method presented in [14] for further validation. Again, the results prove the accuracy of the new proposed IE technique with respect to other different methods. The simulation took 0.9 seconds per frequency point, whereas HFSS ${ }^{\circledR}$ employed 31 seconds, on the same computer as before.

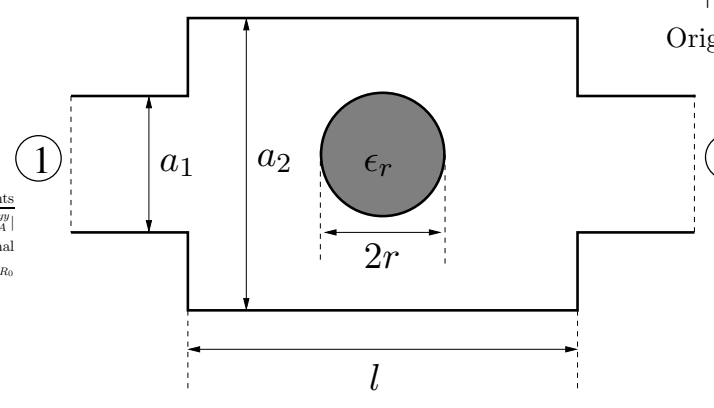

(a) Geometry of a cavity loaded with a dielectric resonator.

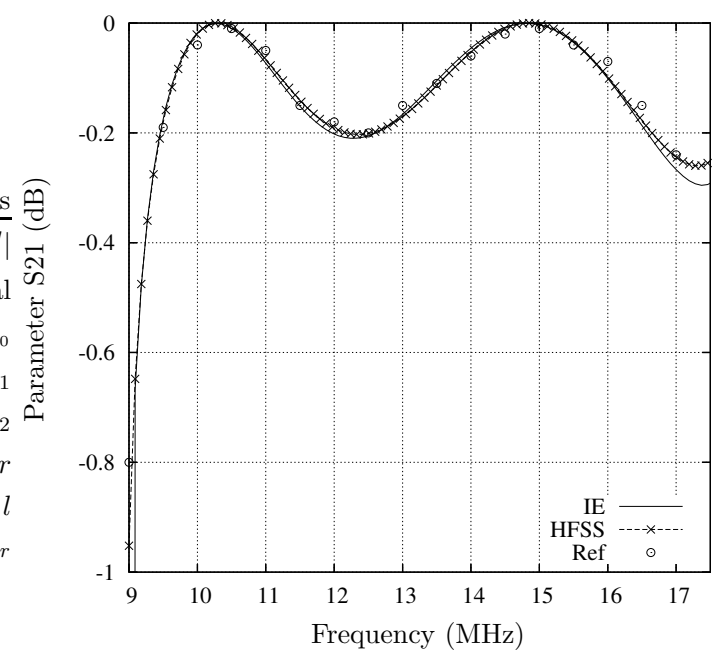

(b) Transmission parameter $\left|S_{21}\right|$ of the structure. Results obtained in [14] (label Ref) are also included for comparison.

Figure 11: Geometry and transmission parameter of a rectangular cavity loaded with a dielectric post placed at the center. Dimensions are: $a_{1}=17.0 \mathrm{~mm}, a_{2}=22.86$ $\mathrm{mm}, r=5 \mathrm{~mm} \mathrm{y} l=30 \mathrm{~mm}$. The relative permittivity of the dielectric post is $\epsilon_{r}=2.17$.

As a third example we present a power divider made in waveguide technology. Fig. 12(a) shows the proposed geometry of the structure, which can be efficiently analyzed with the new double parallel plate method presented in this paper. The obtained scattering parameters are shown in Fig. 12(b), as compared to $\mathrm{HFSS}^{(}{ }^{\circledR}$ results. This structure was modeled with 88 basis functions. In this case 20 modes were needed for the Kummer technique and $P=M=5$ for the Ewald method. Again, convergence is fast since the width of the parallel plate waveguide is small (between 1.136 $\lambda$ and $1.39 \lambda$ for the frequency range of the analysis). The new CAD tool takes only 0.4 seconds per frequency point, whereas $\operatorname{HFSS}^{\circledR}$ needed 76 seconds for the same analysis.

The presented power divider has some interesting features. The usual behavior of a power divider for port \#1 can be observed at the frequency of $9.7 \mathrm{GHz}$. At this frequency the reflection parameter $\left(S_{11}\right)$ has a small value, whereas the transmission parameter to port \#2 $\left(S_{21}\right)$ has a value of $-3 \mathrm{~dB}$. This shows that half of the power is transfered from port \#1 to ports $\# 2$ and \#3. The same device can work in a different way at the frequency of $10.7 \mathrm{GHz}$. At this frequency we introduce the signal 


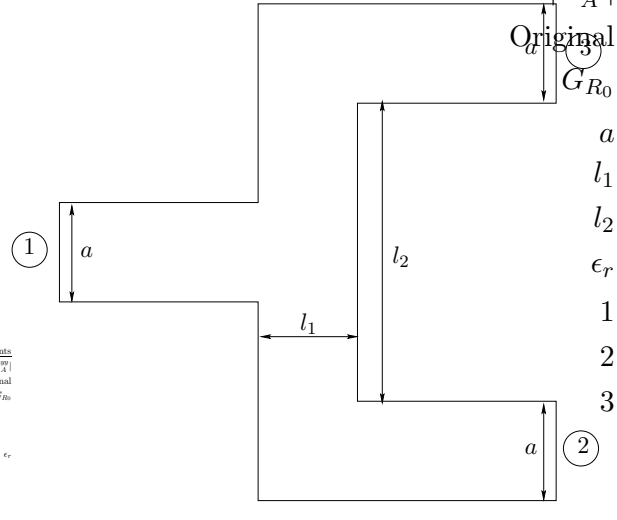

(a) Geometry of the structure.

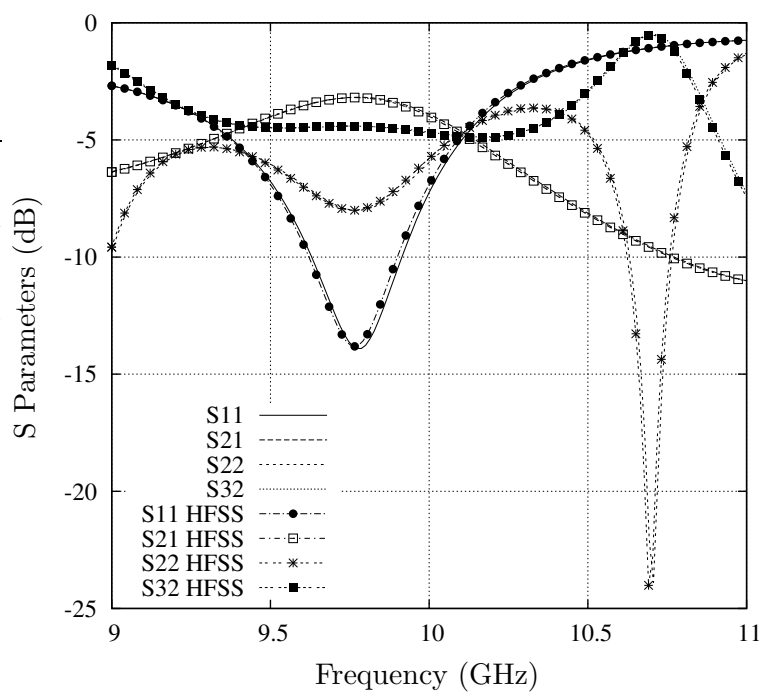

(b) Scattering parameters $(\mathrm{dB})$.

Figure 12: Geometry and scattering parameters of a waveguide power divider. Dimensions are: $a=22.86 \mathrm{~mm}, l_{1}=a, l 2=4 a$.

at port $\# 2$, since the reflection coefficient $\left(S_{22}\right)$ for this port is very small. The signal is essentially transfered from port \#2 to port \#3 $\left(S_{32}=-0.4 \mathrm{~dB}\right)$, whereas a small fraction of the power goes to port \#1 $\left(S_{12}=-9.5 \mathrm{~dB}\right)$. The sample of the signal at port \#1 can be used for monitorization purposes.

The response of the power divider in Fig. 12 can be further enhanced if two dielectric posts are placed symmetrically inside the structure for tuning. The basic dimensions are the same as in the previous example, and two dielectric posts of the same radius are placed as shown in Fig. 13(a). The new response of the structure is presented in Fig. 13(b). The results show a better matching for the



(a) Geometry of the structure.

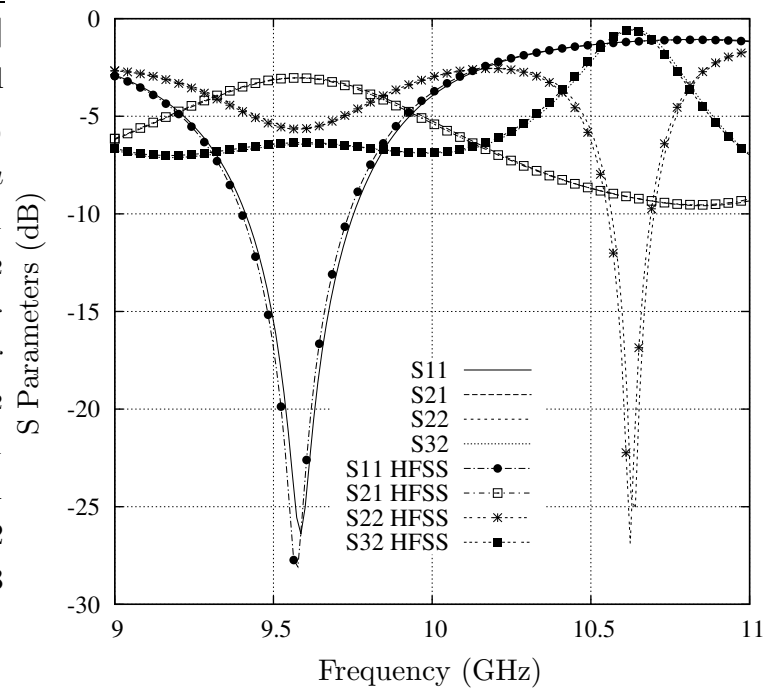

(b) Scattering parameters (dB).

Figure 13: Geometry and scattering parameters of a power divider loaded with two dielectric posts. Dimensions: $a=22.86 \mathrm{~mm} ., l_{1}=a, l 2=4 a$. The radius of the dielectric posts is $r=5 \mathrm{~mm}$, and the distances to the walls are $d_{1}=6.3 \mathrm{~mm}$, and $d_{2}=29.16$ $\mathrm{mm}$. The relative permittivity is $\epsilon_{r}=1.5$ 
power divider, with a deeper reflection coefficient at the frequency of $9.7 \mathrm{GHz}$. The better matching is achieved by adjusting the positions of the dielectric posts. We also observe that the second operational band at $10.7 \mathrm{GHz}$ is hardly affected. Finally, the total frequency response is slightly shifted to higher frequencies with respect to the previous case, due to the influence of the dielectric posts.

In Fig. 13(b), the results obtained with the new approach are again compared with the commercial tool HFSS ${ }^{\circledR}$ to further validate the technique. For the analysis of this compensated power divider, the computational cost was of the same order as for the original divider (for our IE approach, only 48 more basis functions were included to characterize the dielectric posts, taking 0.8 seconds per frequency point). We can observe again good agreement, thus proving the usefulness of the developed technique for the analysis of inductive structures containing dielectric objects.

\section{Conclusions}

In this paper we have presented a new Integral Equation technique based on the Surface Equivalence Principle. The technique combines a surface formulation with the parallel plate waveguide Green's functions, for the analysis of inductive structures containing arbitrarily-shaped conducting and dielectric objects. The technique allows analyzing circuits with an arbitrary number of inputoutput ports, considering that they are aligned along two parallel planes covering the main structure. The most significant feature of the new method is the consideration of two parallel plate waveguides with a 90-degree rotation between their orientations. One of the parallel plates is used for the modeling of the ports. The other one, rotated 90-degrees, generally involves electrically large plate separations, since the whole structure has to fit inside it. A convergence study for the Green's functions series has been presented when the separation of the parallel plates is large. It has been shown that more terms are needed in the series to achieve a given precision, for increasing parallel plate separation. To limit the impact of the degradation of the convergence in the numerical efficiency, a new technique has been proposed. The technique is based on separating the original Green's functions in two terms. The first one is formulated with the slowly convergent series, but it exhibits a very smooth behavior. Therefore, it can be integrated with very few integration points during the calculation of the Method of Moments matrix. The other terms contain the fast variations and the singularity of the Green's functions, and must be treated with special care. However, these terms are formulated with simple Hankel functions, which can be computed very efficiently. Finally, some results have been presented in order to show the accuracy and efficiency of the proposed approach, for analyzing practical multiport inductive waveguide devices.

\section{A Appendix: IE Formulation}

In this appendix we give the details of the integral equation formulated for the analysis of both metallic and dielectric posts. After imposing the proper boundary conditions explained in the theory section, a set of five coupled integral equations is obtained. Using a mixed-potentials formulation 
([15]), these equations are written as follows:

$$
\begin{aligned}
& \hat{n} \times \vec{H}_{n}^{(e x c)}=\hat{n} \times\left\{\sum _ { m = 1 } ^ { N _ { p } } \left[j \omega \int_{S_{p m}} \vec{M}_{p m}\left(\vec{\rho}^{\prime}\right)\left(2 \delta_{m n} \overline{\bar{G}}_{F}^{\left(g p p_{m}\right)}\left(\vec{\rho}, \vec{\rho}^{\prime}\right)+\overline{\bar{G}}_{F}^{\left(g p p_{i n t}\right)}\left(\vec{\rho}, \vec{\rho}^{\prime}\right)\right) d S^{\prime}-\right.\right. \\
& \left.\frac{1}{j \omega} \nabla \int_{S_{p m}} \nabla^{\prime} \cdot \vec{M}_{p m}\left(\vec{\rho}^{\prime}\right)\left(2 \delta_{m n} G_{W}^{\left(g p p_{m}\right)}\left(\vec{\rho}, \vec{\rho}^{\prime}\right)+G_{W}^{\left(g p p_{i n t}\right)}\left(\vec{\rho}, \vec{\rho}^{\prime}\right)\right) d S^{\prime}\right]+ \\
& \frac{1}{\mu_{0}} \nabla \times \int_{S_{c}} \vec{J}_{c}\left(\vec{\rho}^{\prime}\right) \overline{\bar{G}}_{A}^{\left(g p p_{i n t}\right)}\left(\vec{\rho}, \vec{\rho}^{\prime}\right) d S^{\prime}+\frac{1}{\mu_{0}} \nabla \times \int_{S_{d}} \vec{J}_{d}\left(\vec{\rho}^{\prime}\right) \overline{\bar{G}}_{A}^{\left(g p p_{i n t}\right)}\left(\vec{\rho}, \vec{\rho}^{\prime}\right) d S^{\prime}- \\
& \left.j \omega \int_{S_{d}} \vec{M}_{d}\left(\vec{\rho}^{\prime}\right) \overline{\bar{G}}_{F}^{\left(g p p_{i n t}\right)}\left(\vec{\rho}, \vec{\rho}^{\prime}\right) d S^{\prime}+\frac{1}{j \omega} \nabla \int_{S_{d}} \nabla^{\prime} \cdot \vec{M}_{d}\left(\vec{\rho}^{\prime}\right) G_{W}^{\left(g p p_{i n t}\right)}\left(\vec{\rho}, \vec{\rho}^{\prime}\right) d S^{\prime}\right\} ; \quad \text { On } S_{p n} \\
& 0=\hat{n} \times\left\{\sum _ { m = 1 } ^ { N _ { p } } \left[j \omega \int_{S_{p m}} \vec{M}_{p m}\left(\vec{\rho}^{\prime}\right)\left(2 \delta_{m h} \overline{\bar{G}}_{F}^{\left(g p p_{m}\right)}\left(\vec{\rho}, \vec{\rho}^{\prime}\right)+\overline{\bar{G}}_{F}^{\left(g p p_{i n t}\right)}\left(\vec{\rho}, \vec{\rho}^{\prime}\right)\right) d S^{\prime}-\right.\right. \\
& \left.\frac{1}{j \omega} \nabla \int_{S_{p m}} \nabla^{\prime} \cdot \vec{M}_{p m}\left(\vec{\rho}^{\prime}\right)\left(2 \delta_{m h} G_{W}^{\left(g p p_{m}\right)}\left(\vec{\rho}, \vec{\rho}^{\prime}\right)+G_{W}^{\left(g p p_{i n t}\right)}\left(\vec{\rho}, \vec{\rho}^{\prime}\right)\right) d S^{\prime}\right]+ \\
& \frac{1}{\mu_{0}} \nabla \times \int_{S_{c}} \vec{J}_{c}\left(\vec{\rho}^{\prime}\right) \overline{\bar{G}}_{A}^{\left(g p p_{i n t}\right)}\left(\vec{\rho}, \vec{\rho}^{\prime}\right) d S^{\prime}+\frac{1}{\mu_{0}} \nabla \times \int_{S_{d}} \vec{J}_{d}\left(\vec{\rho}^{\prime}\right) \overline{\bar{G}}_{A}^{\left(g p p_{i n t}\right)}\left(\vec{\rho}, \vec{\rho}^{\prime}\right) d S^{\prime}- \\
& \left.j \omega \int_{S_{d}} \vec{M}_{d}\left(\vec{\rho}^{\prime}\right) \overline{\bar{G}}_{F}^{\left(g p p_{i n t}\right)}\left(\vec{\rho}, \vec{\rho}^{\prime}\right) d S^{\prime}+\frac{1}{j \omega} \nabla \int_{S_{d}} \nabla^{\prime} \cdot \vec{M}_{d}\left(\vec{\rho}^{\prime}\right) G_{W}^{\left(g p p_{i n t}\right)}\left(\vec{\rho}, \vec{\rho}^{\prime}\right) d S^{\prime}\right\} ; \quad \text { On } S_{p h} \\
& 0=\hat{n} \times\left\{\sum_{m=1}^{N_{p}}\left[\frac{1}{\epsilon_{0}} \nabla \times \int_{S_{p m}} \vec{M}_{p m}\left(\vec{\rho}^{\prime}\right) \overline{\bar{G}}_{F}^{\left(g p p_{i n t}\right)}\left(\vec{\rho}, \vec{\rho}^{\prime}\right) d S^{\prime}\right]-j \omega \int_{S_{c}} \overline{\bar{G}}_{A}^{\left(g p p_{i n t}\right)}\left(\vec{\rho}, \vec{\rho}^{\prime}\right) \vec{J}_{c}\left(\vec{\rho}^{\prime}\right) d S^{\prime}-\right. \\
& \left.j \omega \int_{S_{d}} \vec{J}_{d}\left(\vec{\rho}^{\prime}\right) \overline{\bar{G}}_{A}^{\left(g p p_{i n t}\right)}\left(\vec{\rho}, \vec{\rho}^{\prime}\right) d S^{\prime}-\frac{1}{\epsilon_{0}} \nabla \times \int_{S_{d}} \vec{M}_{d}\left(\vec{\rho}^{\prime}\right) \overline{\bar{G}}_{F}^{\left(g p p_{i n t}\right)}\left(\vec{\rho}, \vec{\rho}^{\prime}\right) d S^{\prime}\right\} ; \quad \text { On } S_{c} \\
& 0=\hat{n} \times\left\{\sum_{m=1}^{N_{p}}\left[\frac{1}{\epsilon_{0}} \nabla \times \int_{S_{p m}} \vec{M}_{p m}\left(\vec{\rho}^{\prime}\right) \overline{\bar{G}}_{F}^{\left(g p p_{i n t}\right)}\left(\vec{\rho}, \vec{\rho}^{\prime}\right) d S^{\prime}\right]-j \omega \int_{S_{c}} \vec{J}_{c}\left(\vec{\rho}^{\prime}\right) \overline{\bar{G}}_{A}^{\left(g p p_{i n t}\right)}\left(\vec{\rho}, \vec{\rho}^{\prime}\right) d S^{\prime}-\right. \\
& j \omega \int_{S_{d}} \vec{J}_{d}\left(\vec{\rho}^{\prime}\right)\left(\overline{\bar{G}}_{A}^{\left(g p p_{i n t}\right)}\left(\vec{\rho}, \vec{\rho}^{\prime}\right)+G_{A}^{(e l)}\left(\vec{\rho}, \vec{\rho}^{\prime}\right)\right) d S^{\prime}- \\
& \left.\nabla \times \int_{S_{d}} \vec{M}_{d}\left(\vec{\rho}^{\prime}\right)\left[\frac{\bar{G}_{F}^{\left(g p p_{i n t}\right)}\left(\vec{\rho}, \vec{\rho}^{\prime}\right)}{\epsilon_{0}}+\frac{G_{A}^{(e l)}\left(\vec{\rho}, \vec{\rho}^{\prime}\right)}{\epsilon_{1}}\right] d S^{\prime}\right\} ; \quad \text { On } S_{d} \\
& 0=\hat{n} \times\left\{\sum_{m=1}^{N_{p}}\left[j \omega \int_{S_{p m}} \vec{M}_{p m}\left(\vec{\rho}^{\prime}\right) \overline{\bar{G}}_{F}^{\left(g p p_{i n t}\right)}\left(\vec{\rho}, \vec{\rho}^{\prime}\right) d S^{\prime}-\frac{1}{j \omega} \nabla \int_{S_{p m}} \nabla^{\prime} \cdot \vec{M}_{p m}\left(\vec{\rho}^{\prime}\right) G_{W}^{\left(g p p_{i n t}\right)}\left(\vec{\rho}, \vec{\rho}^{\prime}\right) d S^{\prime}\right]+\right. \\
& \frac{1}{\mu_{0}} \nabla \times \int_{S_{c}} \vec{J}_{c}\left(\vec{\rho}^{\prime}\right) \overline{\bar{G}}_{A}^{\left(g p p_{i n t}\right)}\left(\vec{\rho}, \vec{\rho}^{\prime}\right) d S^{\prime}+\frac{1}{\mu_{0}} \nabla \times \int_{S_{d}} \vec{J}_{d}\left(\vec{\rho}^{\prime}\right)\left(\overline{\bar{G}}_{A}^{\left(g p p_{i n t}\right)}\left(\vec{\rho}, \vec{\rho}^{\prime}\right)+G_{A}^{(e l)}\left(\vec{\rho}, \vec{\rho}^{\prime}\right)\right) d S^{\prime}- \\
& j \omega \int_{S_{d}} \vec{M}_{d}\left(\vec{\rho}^{\prime}\right)\left[\overline{\bar{G}}_{F}^{\left(g p p_{i n t}\right)}\left(\vec{\rho}, \vec{\rho}^{\prime}\right) d S^{\prime}+G_{A}^{(e l)}\left(\vec{\rho}, \vec{\rho}^{\prime}\right)\right] d S^{\prime}+ \\
& \left.\frac{1}{j \omega} \nabla \int_{S_{d}} \nabla^{\prime} \cdot \vec{M}_{d}\left(\vec{\rho}^{\prime}\right)\left[G_{W}^{\left(g p p_{i n t}\right)}\left(\vec{\rho}, \vec{\rho}^{\prime}\right)+G_{W}^{(e l)}\left(\vec{\rho}, \vec{\rho}^{\prime}\right)\right] d S^{\prime}\right\} ; \quad \text { On } S_{d}
\end{aligned}
$$


The first equation (3a) is applied to the waveguide corresponding to the exciting port $(n)$, and forces the continuity of the tangential magnetic field on the boundary with the main cavity of the structure $\left(S_{p n}\right)$. The excitation term $\vec{H}_{n}^{(e x c)}$ is produced by a wave propagating in this waveguide (normally the fundamental $T E_{10}$ mode of the waveguide). $\overline{\bar{G}}_{F}^{\left(g p p_{m}\right)}\left(\vec{\rho}, \vec{\rho}^{\prime}\right)$ and $G_{W}^{\left(g p p_{m}\right)}\left(\vec{\rho}, \vec{\rho}^{\prime}\right)$ are, respectively, the dyadic Green's function of the electric vector potential, and the Green's function of the magnetic scalar potential inside the parallel plate waveguide at port $(m)$. Note in this equation the presence of the standard Kronecker delta function between the exciting port $(n)$, and all other ports $(m)$. On the other hand, $\overline{\bar{G}}_{F}^{\left(g p p_{i n t}\right)}\left(\vec{\rho}, \vec{\rho}^{\prime}\right)$ and $G_{W}^{\left(g p p_{i n t}\right)}\left(\vec{\rho}, \vec{\rho}^{\prime}\right)$ are defined in the same way, but for the waveguide defined between the two ground planes in the second equivalent problem (90-degrees rotated parallel plate waveguide). Finally, $\overline{\bar{G}}_{A}^{\left(g p p_{i n t}\right)}\left(\vec{\rho}, \vec{\rho}^{\prime}\right)$ is the dyadic Green's function of the magnetic vector potential of this internal parallel plate waveguide.

Next, equation (3b) is formulated on a port $(h)$ which is not excited $\left(h=1,2 \cdots N_{p} ; h \neq n\right)$. The equation forces the continuity between the tangential magnetic field between port $(h)$ and the internal cavity of the circuit (surface $S_{p h}$ ). The different Green's functions have the same definitions as in the previous equation $(3 \mathrm{a})$.

The third equation (3c) imposes the nullity of the tangential electric field on the conducting surfaces $S_{c}$ (EFIE formulation) for the second problem. The last two equations (3d) and (3e) represent respectively the continuity of the tangential electric and magnetic fields between the second and the third equivalent problems (this is the so-called PMCHWT formulation [7]). $G_{A}^{(e l)}\left(\vec{\rho}, \vec{\rho}^{\prime}\right), G_{A}^{(e l)}\left(\vec{\rho}, \vec{\rho}^{\prime}\right)$ and $G_{W}^{(e l)}\left(\vec{\rho}, \vec{\rho}^{\prime}\right)$ are the corresponding Green's functions of the magnetic and electric vector potentials and magnetic scalar potential, of an infinite unbounded homogeneous region with constitutive parameters $\left(\epsilon_{1}, \mu_{1}\right)$. They are, therefore, formulated as simple Hankel functions.

The set of equations collected in (3) considers a generic conducting surface, and only one dielectric post for the sake of simplicity. The generalization to an arbitrary number of dielectric posts, however, is straightforward. The resulting system of coupled integral equation is then solved by means of the Method of Moments (MoM), employing a standard Galerkin procedure with triangular basis and testing functions. Once the values of the different current densities are obtained, the scattering parameters can be directly obtained by calculating the relation between the incoming wave from the excitation port, and the different reflected waves at each one of the other ports.

\section{References}

[1] S. Yin, T. Valilyeva, and P. Pramanick, "Use of three-dimensional field simulators in the synthesis of waveguide round rod bandpass filters," Int J Microwave and Millimeter Wave Computer Aided Engineering, vol. 8, pp. 484-497, August 1998.

[2] J. M. Reiter and F. Arndt, "Rigorous analysis of arbitrarily shaped H- and E-plane discontinuities in rectangular waveguides by a full-wave boundary contour mode-matching method," IEEE Transactions on Microwave Theory and Techniques, vol. 43, pp. 796-801, April 1995.

[3] T. Rozzi, F. Moglie, A. Morini, W. Gulloch, and M. Politi, "Accurate full-band equivalent circuits of inductive posts in rectangular waveguide," IEEE Transactions on Microwave Theory and Techniques, vol. 40, pp. 1000-1009, May 1992. 
[4] H. Auda and R. F. Harrington, "Inductive posts and diaphragms of arbitrary shape and number in a rectangular waveguide," IEEE Transactions on Microwave Theory and Techniques, vol. 32, pp. 606-613, June 1984.

[5] M. Guglielmi, G. Gheri, M. Calamia, and G. Pelosi, "Rigorous multimode network numerical representation of inductive step," IEEE Transactions on Microwave Theory and Techniques, vol. 42, pp. 317-326, February 1994.

[6] F. Q. Pereira, F. P. Soler, B. G. Martinez, V. E. B. Esbert, J. P. Garcia, J. L. G. Tornero, and A. A. Melcon, "Efficient analysis tool of inductive passive waveguide components and circuits using a novel space domain integral formulation," in European Microwave Conference, (Manchester), IEEE, 11-15, September 2006.

[7] A. J. Poggio and E. K. Miller, Integral Equation Solutions of Three-dimensional Scattering Problems. Oxford: Pergamon Press, 1973.

[8] F. J. P. Soler, F. D. Q. Pereira, J. P. Garcia, D. C. Rebenaque, and A. A. Melcon, "Analysis of inductive waveguide microwave components using an alternative port treatment and efficient fast multipole," Progress In Electromagnetic Research, vol. 68, pp. 71-90, 2007.

[9] A. F. Peterson, S. L. Ray, and R. Mittra, Computational Methods for Electromagnetics, p. 451. IEEE Press, 1998.

[10] W. C. Chew, J.-M. Jin, E. Michielssen, and J. Song, Fast and Efficient Algorithms in Computational Electromagnetics, ch. 2. Artech House, 2001.

[11] Y. Leviatan and G. S. Sheaffer, "Analysis of inductive dielectric posts in rectangular waveguide," IEEE Transactions on Microwave Theory and Techniques, vol. 35, pp. 48-59, January 1987.

[12] F. Capolino, D. R. Wilton, and W. A. Johnson, "Efficient computation of the 2-D Green's function for 1-D periodic structures using the Ewald method," IEEE Transactions on Antennas and Propagation, vol. 53, pp. 2977-2984, September 2005.

[13] W. H. Press, S. A. Teukolsky, W. T. Vetterling, and B. P. Flannery, Numerical Recipes in Fortran 90, The Art of Parallel Scientific Computing. The Pitt Building, Trumpignton Street, Cambridge: Cambridge University Press, 1996.

[14] D. Camilleri, B. Gimeno, A. Coves, M. V. Andres, A. A. Blas, H. Esteban, and V. E. Boria, "Efficient technique for the analysis of cylindrical dielectric resonators in rectangular waveguide cavities," in Proceedings of PIERS 2002, p. 617, PIER, July 2002.

[15] J. R. Mosig, Integral Equation Technique. New York: Wiley Interscience Publication, 1989. 